Discussion Paper No. 02-29

\title{
Climate Change and the
} Irreversibility Effect -

Combining Expected Utility and MaxiMin

Andreas Lange

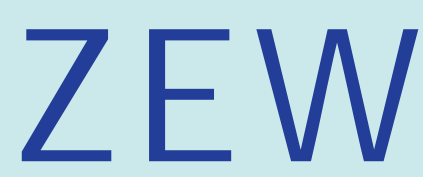

Zentrum für Europäische Wirtschaftsforschung $\mathrm{GmbH}$

Centre for European Economic Research 
Discussion Paper No. 02-29

\title{
Climate Change and the Irreversibility Effect - \\ Combining Expected Utility and MaxiMin
}

\author{
Andreas Lange
}

Download this ZEW Discussion Paper from our ftp server:

\author{
ftp://ftp.zew.de/pub/zew-docs/dp/dp0229.pdf
}

Die Discussion Papers dienen einer möglichst schnellen Verbreitung von neueren Forschungsarbeiten des ZEW. Die Beiträge liegen in alleiniger Verantwortung der Autoren und stellen nicht notwendigerweise die Meinung des ZEW dar.

Discussion Papers are intended to make results of ZEW research promptly available to other economists in order to encourage discussion and suggestions for revisions. The authors are solely responsible for the contents which do not necessarily represent the opinion of the ZEW. 


\section{Non-technical summary}

The impact of anthropogenic greenhouse gas emissions is subject to substantial uncertainties which could be reduced by future learning. One view is that cost-intensive measures should be postponed until better information is available. The contrary view is that, because of the possibility of learning, one should pursue even stricter abatement policies today in order not to irreversibly accumulate long-lived greenhouse gases in the atmosphere ("irreversibility effect").

While expected utility maximization (EU) is the most common decision criterion under uncertainty, one may doubt the applicability of this concept to the problem of future climate change: First, there is no objective probability assessment. Secondly, empirical evidence indicates that, quite regularly, people do not maximize EU, particularly if extreme outcomes are involved.

In this paper, I therefore analyze the irreversibility effect when people give more weight to the worst case than standard expected utility (EU) maximizers do. Such a combination of EU and the MaxiMin criterion was suggested in the literature but has so far only been applied to static, not to intertemporal problems.

I first study the effects of an increase of confidence, i.e. of a move from MaxiMin to EU, on first period emission levels. Contrary to the intuitive results from the static models, such increased confidence does not necessarily lead to an expansion of optimal emissions. Analyzing the consequences of incorporating the prospective new information into today's decisions, I demonstrate that standard results from EU on the irreversibility effect are fairly fragile to the weight attached to MaxiMin: For quadratic utility functions the effects of learning are reversed if one moves away from EU. In other words, whereas learning leads to higher emissions and the irreversibility effect does not apply for high confidence levels (criteria close to EU), the irreversibility effect holds for small levels of confidence in the probability assessment.

There is, however, the possibility of a negative value of learning. For quadratic utility functions, it is shown that the irreversibility effect holds if and only if the value of learning is negative. Taking the extended EU - MaxiMin criterion as a descriptive model, this would explain if people who attach a large weight on the worst case choose not to receive new information on the prospective damages. Consequences for the applicability of generalized EU-MaxiMin are also discussed. 


\title{
Climate change and the irreversibility effect - Com- bining Expected Utility and MaxiMin
}

\author{
Andreas Lange \\ Interdisciplinary Institute for Environmental Economics, University of Heidelberg \\ Centre for European Economic Research (ZEW), Mannheim
}

\begin{abstract}
This paper analyzes decisions on emissions of a stock pollutant under uncertainty in a two period model. Decisions are based on a weighted average of expected utility (EU) and the MaxiMin criterion. I first show that more weight on the worst case (less weight on EU) may lead to increased first period emissions. The effect of learning possibilities on emissions is not clear in general, but depends qualitatively on the weight given to MaxiMin: For the quadratic utility case, considering prospective learning increases today's abatement effort, i.e. the "irreversibility effect" holds, if the weight on EU is small. This contrasts standard results on the irreversibility effect for EU which translates to small weights on MaxiMin. There is, however, the possibility of a negative value of learning. It is shown that the irreversibility effect holds if and only if the value of learning is negative. Consequences for the applicability of generalized EU-MaxiMin are discussed.
\end{abstract}

JEL: D81, D60, H4

Keywords: uncertainty, MaxiMin, irreversibility, learning

\section{Correspondence:}

A. Lange, Centre for European Economic Research (ZEW)

P.O.Box 103443, D-68034 Mannheim, Germany

Phone: (++49) 621 - 1235 208, Fax: 1235226

Email: lange@zew.de, lange@uni-hd.de 


\section{Introduction}

The impact of anthropogenic greenhouse gas emissions is subject to substantial uncertainties which could be reduced by future learning. In this paper, I analyze how this potentially better information in the future affects today's choice of greenhouse gas emissions when people give more weight to the worst case than standard expected utility (EU) maximizers do. For such a combination of EU and the MaxiMin criterion, I show that standard results on the effect of learning crucially depend on the weight given to the worst case.

The criterion most prevailing in economic literature on decisions under uncertainty is expected utility maximization (EU) which relies on a single (objective or subjective) probability distribution. For the problem of future climate change, however, one may doubt the applicability of this concept. First, there is no objective probability assessment. Although this is mentioned in the IPCC report ${ }^{1}$ (IPCC III 1996:65), it is even better illustrated by a study by Morgan and Keith (1995). They state probability assessments of 16 climate experts for various climate-change-related aspects which "indicate a greater disagreement than we believe is usually conveyed in scientific consensus documents" (Morgan and Keith 1995:468A). Secondly, empirical evidence indicates that, quite regularly, people do not maximize EU, in particular if extreme low-probability outcomes are involved. ${ }^{2}$

A decision criterion sometimes suggested in the literature (recently by Bretteville 1999, in similar form Chichilnisky 2000) is to put more weight on the worst case, thereby being more sensitive to low probability catastrophic events. In other words, one maximizes a weighted sum of expected utility and the utility in the worst case scenario. ${ }^{3}$ That is, while there is some probability assessment, the confidence in this distribution is limited. Here, for example, the probabilities may stem from an aggregation of several experts' judgments. ${ }^{4}$ The weight attached to the expected utility maximization can thereby

\footnotetext{
${ }^{1} \mathrm{IPCC}=$ Intergovernmental Panel on Climate Change which assesses the scientific, technical and socio-economic information relevant for the understanding of human-induced climate change.

${ }^{2}$ For example, the behavior according to the Ellsberg-paradox (Ellsberg 1961) is on conflict with EU. An overview on alternative decision criteria which can incorporate such deviations from EU is given by Camerer and Weber (1992).

${ }^{3}$ This criterion can be understood as a special case of the axiomized Choquet-expected utility theory which has been developed by Gilboa (1987) and Schmeidler (1989). See for example Eichberger and Kelsey (1997) for an introduction to this theory.

${ }^{4}$ As mentioned by Morgan and Keith (1995), consensus methods like the Delphi method could be applied in order to come up with some aggregated assessment. Alternatively, a decision maker could
} 
be interpreted as the confidence of the decision maker into the aggregated probability assessment. To put it differently, the weight given to the worst case is a measure for the degree of uncertainty.

Bretteville (1999) and Chichilnisky (2000) study the weighted EU and MaxiMin criterion in a static context. Here, less confidence in the probability assessment generally leads to increased abatement effort, i.e. less emissions. However, the nature of the climate change problem suggests a dynamic approach which is taken in this paper: On the one hand, uncertainty might be resolved over time. On the other hand, since greenhouse gases accumulate in the atmosphere, the emissions are irreversible up to the specific lifetime of greenhouse gases (eg. 120 years for $\mathrm{CO}_{2}$ ) and therefore the stock cannot be reduced even if new information shows that global warming leads to severe damages.

From a theoretical perspective, this issue of irreversibility of decisions has been addressed by Arrow and Fisher (1974), Henry (1974), Chichilnisky and Heal (1993) and others. The basic finding is that making an irreversible decision induces some costs (quasi-option value) because no change of decision is possible in light of new information. Thus, if there is the possibility of additional future information it gets more important to keep future options open, i.e. taking a more flexible position. With respect to the greenhouse gas problem, this would imply that current emission levels should be lower if we can learn more about damages in the future (referred to as "irreversibility effect").

However, as shown by Ulph and Ulph (1997) within a simple EU-model of global warming (based on Epstein 1980), the irreversibility effect does not necessarily apply to the greenhouse gas emissions. In their simulations, the possibility of future learning leads to increased current period emissions for most parameters. For quadratic utility functions, they even show that the irreversibility effect never holds. The reason is that more flexibility with respect to the stock of greenhouse gases by pursuing stricter abatement policies involves higher abatement costs.

In this paper, I analyze the irreversibility effect and explore the consequences of applying the weighted EU and Maximin criterion in a two period model. By analogy to Ulph and Ulph (1997), decisions have to be made on greenhouse gas emissions and accumulate in the atmosphere. The stock of gases causes environmental damages which are uncertain at the beginning of period 1. New information on the harmfulness arrives,

just middle the experts views by some technique. 
however, before the start of the second period, albeit before second period emission level is fixed. Thus, second period emissions may be contingent on the received signal. However, applying the weighted EU-MaxiMin criterion, this contingency may lead to dynamically inconsistent decisions. That is, after receiving the signal and updating the beliefs, the optimal decision may not be consistent with the emission level that was considered to be optimal from an ex ante point of view. ${ }^{5}$ To overcome this problem, the concept of behaviorally consistency was introduced by Karni and Safra (1989). This concept - which I apply in this paper - allows to calculate optimal decisions by standard backward induction.

I first study the effects of an increase of confidence, i.e. of a move from MaxiMin to EU, on first period emission levels. Contrary to the intuitive results from the static models, such increased confidence does not necessarily lead to an expansion of optimal emissions. Analyzing the consequences of incorporating the prospective new information into today's decisions, I demonstrate that Ulph and Ulph's result on the irreversibility effect is fairly fragile to the weight attached to MaxiMin: For quadratic utility functions, if one moves away from EU, the effects of learning are reversed. In other words, whereas for high confidence levels, that is decision criteria close to expected utility maximization, learning leads to higher emissions and the irreversibility effect does not apply (Ulph and Ulph 1997), for small levels of confidence in the probability assessment it is optimal to emit less than one would do if no learning were possible. Here, the irreversibility effect holds.

As will be shown, the irreversibility effect can be related to the value of (costless) learning, i.e. the difference in ex ante expected utility if future learning is / is not taken into account. Due to deviations from EU, this value can be negative (see Wakker 1988). For quadratic utility functions it is shown that the irreversibility effect holds if and only if the value of learning is negative. Taking the extended EU - MaxiMin criterion as a descriptive model, this would explain uncertainty averse people (with low confidence into the probability assessment) who choose not to receive new information on the prospective damages. Such people would be afraid of getting an information which indicates relative harmlessness of emissions whereas finally severe damages result. Such an information would induce them to choose a higher emission level in period 2 than if learning would not be possible. Thus, if the information cannot be disregarded, they would obviate this potential mistake of building up a too large stock by emitting less in period 1 .

\footnotetext{
${ }^{5}$ It was shown by Epstein and le Breton (1993) and Machina (1989) that this problem generally may emerge when a non-expected utility framework is applied.
} 
The remainder of the paper is organized as follows. In section 2 , I present the basic model and study the general first order conditions in section 2.1. In section 2.2 the effect of the confidence parameter, in section 2.3 the effect of learning is analyzed. The final section 3 concludes.

\section{The model}

Consider a two period model with stock pollution. Emissions, $e_{1}$ in period 1 and $e_{2}$ in period 2, yield flow benefits $V^{l}\left(e_{l}\right)$ in period $l$ (increasing and concave), whereas the stock at the end of period 2 causes some damage $D\left(e_{1}+e_{2}, s\right)$ (increasing and convex in e) which depends on a parameter $s \in \mathcal{S}$ which captures the uncertainty at the start of period 1. It is assumed that damage and marginal damage increase in $s$, i.e. $s$ can be interpreted as a damage parameter. Thus, the total payoff to the representative consumer is specified as

$$
u=U\left(e_{1}, e_{2}, s\right)=V^{1}\left(e_{1}\right)+V^{2}\left(e_{2}\right)-D\left(e_{1}+e_{2}, s\right){ }^{6}
$$

I assume that after the first period, albeit before decisions on second period emissions have to be fixed, new information on the state of nature, i.e. the expected damages, may arrive. The set of possible signals, $\mathcal{I}$, is assumed to be finite. A tuple $(i, s) \in \mathcal{I} \times \mathcal{S}$ is referred to as a state of the world. Obviously, the decision maker can condition her second period decisions on the new information, i.e. $e_{2}=e_{2}(i)$. In this paper I study and compare two cases: The decision maker (i) could or (ii) could not take into account the prospective new information when deciding about the first period emission level. These cases are referred to as the "learning"-case "L" and the "non-learning" case "N", respectively.

The decision criterion analyzed in this paper gives more weight to the worst case than a standard expected utility framework. Assuming that expected utility is based on a probability distribution $\mu(i, s)$, ex ante welfare is given by

$$
\lambda \int_{\mathcal{I} \times \mathcal{S}} U\left(e_{1}, e_{2}(i), s\right) d \mu(i, s)+(1-\lambda) \min _{(i, s)} U\left(e_{1}, e_{2}(i), s\right)
$$

\footnotetext{
${ }^{6}$ Note that under our assumptions $s \geq \bar{s}$ implies both, $U\left(e_{1}, e_{2}, s\right) \leq U\left(e_{1}, e_{2}, \bar{s}\right)$ and $U_{e_{l}}\left(e_{1}, e_{2}, s\right) \leq$ $U_{e_{l}}\left(e_{1}, e_{2}, \bar{s}\right)$ for $l=1,2$, i.e. a larger damage parameter $s$ implies both, less utility and a reduced marginal utility.
} 


\subsection{The optimal choice of emission levels}

In the case "N" without learning, $e_{2}$ clearly does not depend on the signal $i$. Hence, the worst case is determined solely by the maximal damage parameter max $s$ and the optimal decision $\left(e_{1}^{N}, e_{2}^{N}\right)$ is given by the first-order-condition

$$
0=\lambda \int_{\mathcal{S}} U_{e_{l}}\left(e_{1}, e_{2}, s\right) d \mu(s)+(1-\lambda) U_{e_{l}}\left(e_{1}, e_{2}, \max s\right) \text { for } l=1,2
$$

A "rational" decision maker, however, conditions her $e_{2}$-choice on the observed signal. For the weighted EU - MaxiMin criterion, this may lead to temporally inconsistent decisions: If the emission plan $\left(e_{1},\left(e_{2}(i)\right)_{i}\right)$ maximizes ex ante preferences $(2)$, the decision maker in general will deviate from this plan after receiving a signal $i$. This inconsistency problem - which arises for all decision criteria that deviate from EU (Machina 1989) - can be solved by concentrating on those emission plans that will be stuck to after receiving the new information. ${ }^{7}$ In other words, given a signal $i$ and a first period emission level $e_{1}$, optimal second period emissions, denoted by $e_{2}^{L}\left(e_{1}, i\right)$, maximize the updated preferences

$$
\begin{aligned}
& \lambda \int_{\mathcal{S}} U\left(e_{1}, e_{2}, s\right) d \mu(s \mid i)+(1-\lambda) \min _{s} U\left(e_{1}, e_{2}, s\right) \\
= & \lambda \int_{\mathcal{S}} U\left(e_{1}, e_{2}, s\right) d \mu(s \mid i)+(1-\lambda) U\left(e_{1}, e_{2}, \max s\right)
\end{aligned}
$$

The first order condition is given by

$$
0=\lambda \int_{\mathcal{S}} U_{e_{2}}\left(e_{1}, e_{2}^{L}\left(e_{1}, i\right), s\right) d \mu(s \mid i)+(1-\lambda) U_{e_{2}}\left(e_{1}, e_{2}^{L}\left(e_{1}, i\right), \max s\right)
$$

This choice of $e_{2}^{L}\left(e_{1}, i\right)$ determines the ex ante worst case, i.e. the state of the world which leads to the smallest utility and is given more weight. Note that the two period structure implies that the worst case corresponds to a tupel $(i, s)$. This worst perceivable case occurs if the information $i$ leads to maximal second period emissions $\max _{i} e_{2}^{L}\left(e_{1}, i\right)$, but finally the worst state of nature $\max s$ is realized. This directly follows from equation (5) and the assumption that marginal utility decreases in $s$. To see this, note that

$$
U_{e_{2}}\left(e_{1}, e_{2}^{L}\left(e_{1}, i\right), \max s\right) \leq 0 .
$$

Hence we obtain for all $i \in \mathcal{I}$ and $s \in \mathcal{S}$

$$
\left.U\left(e_{1}, e_{2}^{L}\left(e_{1}^{L}, i\right), s\right) \geq U\left(e_{1}, e_{2}^{L}\left(e_{1}, i\right), \max s\right) \geq U\left(e_{1}, \max _{j} e_{2}^{L}\left(e_{1}, j\right)\right), \max s\right) .
$$

\footnotetext{
${ }^{7}$ This solution concept of behavioral consistency was introduced by Karni and Safra (1989).
} 
Thus, the worst thing that can happen from an ex ante point of view is that the damage from emissions is maximal although the information suggested emissions to be relatively harmless. The optimal emission plan therefore solves

$$
\max _{e_{1}}\left[\lambda \int_{\mathcal{I} \times \mathcal{S}} U\left(e_{1}, e_{2}^{L}\left(e_{1}, i\right), s\right) d \mu(i, s)+(1-\lambda) U\left(e_{1}, \max _{i} e_{2}^{L}\left(e_{1}, i\right), \max s\right)\right],
$$

and results in the first order condition

$$
\begin{gathered}
0=\lambda \int_{\mathcal{I} \times \mathcal{S}}\left[U_{e_{1}}\left(e_{1}, e_{2}^{L}\left(e_{1}, i\right), s\right)+U_{e_{2}}\left(e_{1}, e_{2}^{L}\left(e_{1}, i\right), s\right) \frac{\partial e_{2}^{L}\left(e_{1}, i\right)}{\partial e_{1}}\right] d \mu(i, s) \\
+(1-\lambda)\left[U_{e_{1}}\left(e_{1}, \max _{i} e_{2}^{L}\left(e_{1}, i\right), \max s\right)\right. \\
\left.+U_{e_{2}}\left(e_{1}, \max _{i} e_{2}^{L}\left(e_{1}, i\right), \max s\right) \frac{\partial \max e_{2}^{L}\left(e_{1}, i\right)}{\partial e_{1}}\right]
\end{gathered}
$$

This optimal emission plan $\left(e_{1}^{L},\left\{e_{2}^{L}\left(e_{1}^{L}, i\right)\right\}_{i}\right)$ does not maximize the ex ante preferences, although it is the best emission plan that the decision maker would stick to after receiving new information. This discrepancy between ex ante preferences and the choice of second period emissions based on updated preferences is indicated by the fact that - differently from expected utility maximization - the partial derivatives with respect to $e_{2}(i)$ do not cancel out. To see this, note that condition (5) implies that

$$
\int_{\mathcal{S}} U_{e_{2}}\left(e_{1}, e_{2}^{L}\left(e_{1}, i\right), s\right) d \mu(s \mid i) \geq 0
$$

on the one hand, but

$$
U_{e_{2}}\left(e_{1}, e_{2}^{L}\left(e_{1}, i\right), \max s\right) \leq 0
$$

on the other. Plugging this into (7), from the ex ante view, second period emissions $e_{2}^{L}\left(e_{1}, i\right)$ for $i \notin \operatorname{argmax}_{j} e_{2}^{L}\left(e_{1}, j\right)$ are chosen at a level too low. ${ }^{8}$ Since first and second period emission levels are negatively correlated $\left(\partial e_{2}^{L}\left(e_{1}, i\right) / \partial e_{1}\right)$, this discrepancy tends to decrease $e_{1}^{L}$. For $i \in \operatorname{argmax}_{j} e_{2}^{L}\left(e_{1}, j\right)$, however, the emission level is too high from the ex ante point of view, in tendency increasing $e_{1}$.

After having described how the decision maker finds the optimal emission level, in the following sections the role of the confidence parameter $\lambda$ for decisions and the role of learning is analyzed.

\footnotetext{
${ }^{8}$ Given $e_{1}$, maximization of (2) with respect to $e_{2}(i)$ would yield $\int_{\mathcal{S}} U_{e_{2}}\left(e_{1}, e_{2}(i), s\right) d \mu(s \mid i)=0$, and, therefore, $e_{2}(i)>e_{2}^{L}\left(e_{1}, i\right)$
} 


\subsection{The impact of the confidence parameter}

Let us first study the impact of the confidence parameter on the optimal emission levels in the case "N". Here, in line with the results in static models (e.g. Asheim and Bretteville 2001), the effect is easily seen from (3): The larger $\lambda$, the less weight is given to the worst case, i.e. to the maximal damage. Thus, marginal damage decreases and first period emissions $e_{1}^{N}$ are increased, and analyze the partial derivative of (7) with respect to $\lambda$.

For the "L"-case, however, this is different. To get an intuition on the differences, let us consider only the direct effect of $\lambda$ on the first order condition (7), i.e. we assume for the moment that $e_{2}^{L}(\cdot, i)$ are fixed.

Then, on the one hand, there is a similar effect as in the " $\mathrm{N}$ " case: By shifting the weight away from the worst case, marginal utility of first period emissions emissions is increased

$$
\int U_{e_{1}}(\cdot, \cdot, s) d \mu(i, s)-U_{e_{1}}(\cdot, \cdot, \max s)>0
$$

Thus, first period emissions could be raised.

On the other hand, however, we have already noted that - from the ex ante view second period emissions are too low for $i \notin \operatorname{argmax}_{j} e_{2}^{L}\left(e_{1}, j\right)$ (tending to decrease $e_{1}$ ), whereas they are too high for $i \in \operatorname{argmax}_{j} e_{2}^{L}\left(e_{1}, j\right)$ (implying a larger $e_{1}$ ). For an increase in $\lambda$, this discrepancy now is weighted more in the former case, less in the latter case:

$$
\left[\int U_{e_{2}}(\cdot, s) \frac{\partial e_{2}^{L}\left(e_{1}, i\right)}{\partial e_{1}} d \mu(i, s)\right]-U_{e_{2}}(\cdot, \max s) \frac{\partial \max e_{2}^{L}\left(e_{1}, i\right)}{\partial e_{1}}<0
$$

Consequently, this effect tends to decrease first period emissions. In addition, clearly also second period emissions $e_{2}^{L}(\cdot, i)$ change with $\lambda$.

The sum of all effects has an ambiguous sign. That is, an increase of the confidence parameter $\lambda$ has an ambiguous impact on emissions $e_{1}^{L}$ in period 1 . This claim is illustrated by the following example:

Example 1 Let the utility function be given by

$$
U\left(e_{1}, e_{2}, s\right)=-\left(1-e_{1}\right)^{2}-\left(1-e_{2}\right)^{2}-s\left(e_{1}+e_{2}\right)^{2}
$$

The damage parameter $s$ may take the values 0,1 , i.e. $\max s=1$. Let us assume that two signal $i=A, B$ are possible with $\mu(A)=0.1, \mu(B)=0.9$ where $s^{A}=\int s d \mu(s \mid$ 
$A)=0, s^{B}=\int s d \mu(s \mid B)=1$. Then, as derived in the appendix, the optimal emission level in period 1 is given by

$$
e_{1}^{L}=\frac{1-\left[\lambda \int\left(N(i)^{2}+s^{i}\right) /(1+N(i))^{2} d \mu(i)+(1-\lambda)\left(N(i)^{2}+\max s\right) /(1+N(i))^{2}\right]}{1+\lambda \int\left(N(i)^{2}+s^{i}\right) /(1+N(i))^{2} d \mu(i)+(1-\lambda)\left(N(i)^{2}+\max s\right) /(1+N(i))^{2}}
$$

where $N(i)=\lambda s^{i}+(1-\lambda) \max s$.

For this example, first period emissions increase for small $\lambda$ then decrease and finally rise again. The emission level as a function of the confidence is illustrated in figure 1.

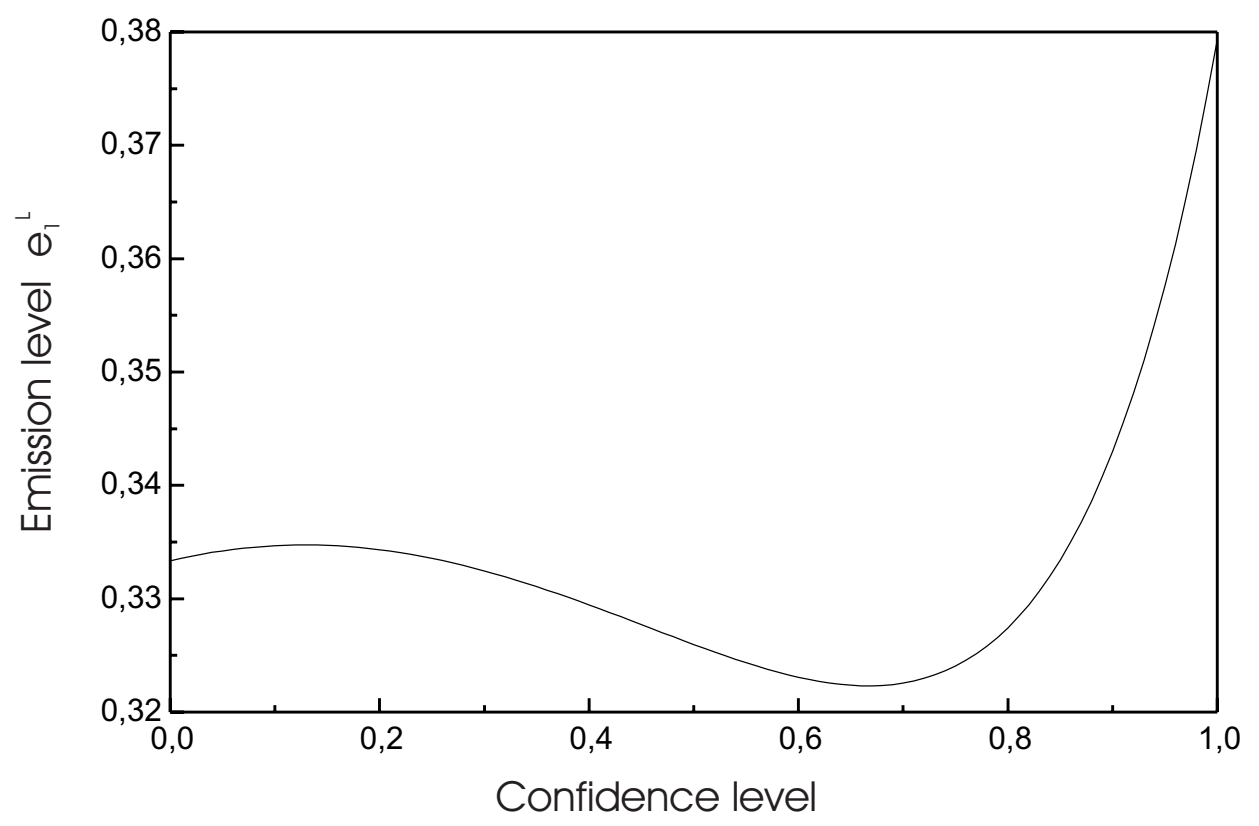

Figure 1: $e_{1}^{L}$ as a function of the confidence level $0 \leq \lambda \leq 1$.

Thus, if the decision maker maximizes the weighted average of EU and worst case in both periods, shifting the weight towards EU does not necessarily imply an increase of emissions as one might expect if less weight is given to the worst case scenario. This reverses the intuitive findings for the non-learning case and the static model (e.g. Aaheim and Bretteville 2001). Rather, it was shown by the example that the optimal emission level may decrease in $\lambda$.

\subsection{The effect of learning}

In this section, let us study the effect of learning on first period decisions, that is the differences in first period emission levels between the cases with and without learning. 
Here, the qualitative effects of learning crucially depend on the confidence level $\lambda$ which is attached to EU. In other words, conclusions on the irreversibility effect from expected utility maximization are fairly fragile to a slight change in the decision criterion. To demonstrate this, I concentrate on the case of quadratic utility functions. On the one hand, these can be interpreted as an approximation of an arbitrary function; on the other hand, they build a clear-cut benchmark since Ulph and Ulph (1997) have shown that the irreversibility effect does not hold for quadratic functions if EU is applied.

Assumption 1 Marginal utility and marginal damage are given by

$$
\begin{aligned}
V_{e_{1}}^{1}\left(e_{1}\right) & = \begin{cases}a_{11}-a_{12} e_{1} & \text { for } 0 \leq e_{1}<a_{11} / a_{12} \\
0 & \text { for } e_{1}>a_{11} / a_{12}\end{cases} \\
V_{e_{2}}^{2}\left(e_{2}\right) & = \begin{cases}a_{21}-a_{22} e_{2} & \text { for } 0 \leq e_{2}<a_{21} / a_{22} \\
0 & \text { for } e_{2}>a_{21} / a_{22}\end{cases} \\
D_{e}(e, s) & =\left(b_{1}+s b_{2}\right)\left(E_{0}+e\right)
\end{aligned}
$$

where $a_{l t}, b_{t}, E_{0} \geq 0(l, t=1,2)$ and, further, $s<\bar{s}$, guaranteeing positive emission levels in both periods.

Note that $E_{0}$ can be interpreted as the stock of greenhouse gases which already exists at the beginning of period $1 .^{9}$ A sufficient upper bound $\bar{s}$ for the damage parameter and the optimal emission levels are derived in the appendix. Clearly, $e_{1}^{x}(x=L, N)$ depend on the confidence parameter $\lambda$. Their functional form is given by

$$
\begin{aligned}
e_{1}^{x} & =\frac{a_{11}-\left[a_{21}+a_{22} E_{0}\right] K^{x}}{a_{12}+a_{22} K^{x}} \\
\text { where } K^{x} & =\lambda \int k\left(N(i), s^{i}\right) d \mu(i)+(1-\lambda) k\left(\min _{i} N(i), \max s\right)
\end{aligned}
$$

where $k(\cdot, \cdot)$ is defined in (14) in the appendix and $N(i)=\lambda \int s d \mu(s \mid i)+(1-\lambda) \max s$ for the "L"-case and $N(i)=\bar{N}:=\lambda \int s d \mu(s)+(1-\lambda) \max s$ for $x=N$.

To analyze how the effect of learning on first period decisions, that is the differences in first period emission levels between the cases with and without learning, depends on the confidence level, we compare (9) for $x=N, L$ for different $\lambda$.

\footnotetext{
${ }^{9}$ In particular, the first unit of anthropogenic greenhouse gas would cause no harm $\left(D_{e}(0, s)=0\right.$ if $\left.E_{0}=0\right)$.
} 
For $\lambda=1$, one is obviously back to EU. As already mentioned by Ulph and Ulph (1997), using the quadratic specification of the utility function the consideration of learning possibilities leads to an increase of emissions. Hence, the irreversibility effect does not hold. Thus, due to continuity, the same will hold true for large confidence levels $\lambda$.

For $\lambda=0$, i.e. the MaxiMin criterion, learning has no effect since in both periods only the worst case matters and second period emissions do not depend on the signal which is received before the start of period 2 .

For small confidence levels $\lambda$, i.e. if much weight is given to the worst case, however, the result of EU is reversed:

\section{Proposition 1 Let the utility function satisfy assumption 1.}

If the ex ante confidence in the probability assessment is low, i.e. close to zero, then the irreversibility effect holds. That is, first period emission levels are lower in the learning case, i.e. $e_{1}^{L}<e_{1}^{N}$.

To prove proposition 1 note again that learning has no effect on emissions if the uncertainty is maximal, i.e. $\lambda=0 .{ }^{10}$ We therefore have to study the derivatives of $e_{1}^{L}$ and $e_{1}^{N}$ with respect to $\lambda$ at $\lambda=0$. Whereas the first derivatives with respect to $\lambda$ coincide, the second derivatives satisfy the claimed relationship. The complete proof is given in the appendix. ${ }^{11}$

Proposition 1 states that if the degree of uncertainty is high, the irreversibility effect with respect to the stock of emissions leads to smaller first period emissions when learning possibilities are taken into account. Thus, the standard result obtained from expected utility maximization is reversed for large levels of uncertainty. I.e., the implications of learning capacities on today's decision do heavily depend on the confidence the decision maker has into the probability assessment. If the uncertainty aversion with respect to environmental damage is very large, then more weight is given to the irreversibility of damage due to climate change, and learning leads to lower emissions. If the uncertainty is considered to be small, then the monetary irreversibility of capital expenditures to reduce $\mathrm{CO}_{2}$ emissions dominates and less strict abatement policies would be chosen.

\footnotetext{
${ }^{10}$ This follows immediately from $(9)$ since $N(i)=\max s$ for the "L" and the "N" case.

${ }^{11}$ At $\lambda=0$, the first derivative of (9) w.r.t. $\lambda$ are $\int k\left(N(i), s^{i}\right) d \mu(i)-k\left(\min _{i} N(i), \max s\right)$. Again, $N(i)=\max s$ for both the "L"- and the " $\mathrm{N}$ "-case and therefore the first derivatives coincide. For the second derivatives, we have to consider $\frac{\partial k(\cdot, \cdot)}{\partial N(i)}$ which is done in the appendix.
} 
The following example illustrates the claim of proposition 1:

Example 2 Let the utility function again be given by

$$
U\left(e_{1}, e_{2}, s\right)=-\left(1-e_{1}\right)^{2}-\left(1-e_{2}\right)^{2}-s\left(e_{1}+e_{2}\right)^{2}
$$

where $s$ can take the values 0 and 1 . Two signals $i=A, B$ can be received with ex ante probabilities $\mu(A)=0.1$ and $\mu(B)=0.9$, respectively. The conditional probabilities are assumed to be given by

$$
\mu(0 \mid A)=0.75=1-\mu(1 \mid A) \text { and } \mu(0 \mid B)=0.25=1-\mu(1 \mid B)
$$

In figure 2 we show the impact of the degree of confidence on optimal decisions. Here, the difference between first period emissions in the "learning" and the "non-learning" case, $e_{1}^{L}-e_{1}^{N}$, is depicted as functions of $\lambda \in[0,1]$. The picture illustrates the wellknown fact that $e_{1}^{L}$ exceeds $e_{1}^{N}$ for expected utility maximization $(\lambda=1)$. However, this relationship is reversed over a wide range of confidence levels. Thus, in our example the irreversibility effect holds for most weights given to EU or MaxiMin respectively.

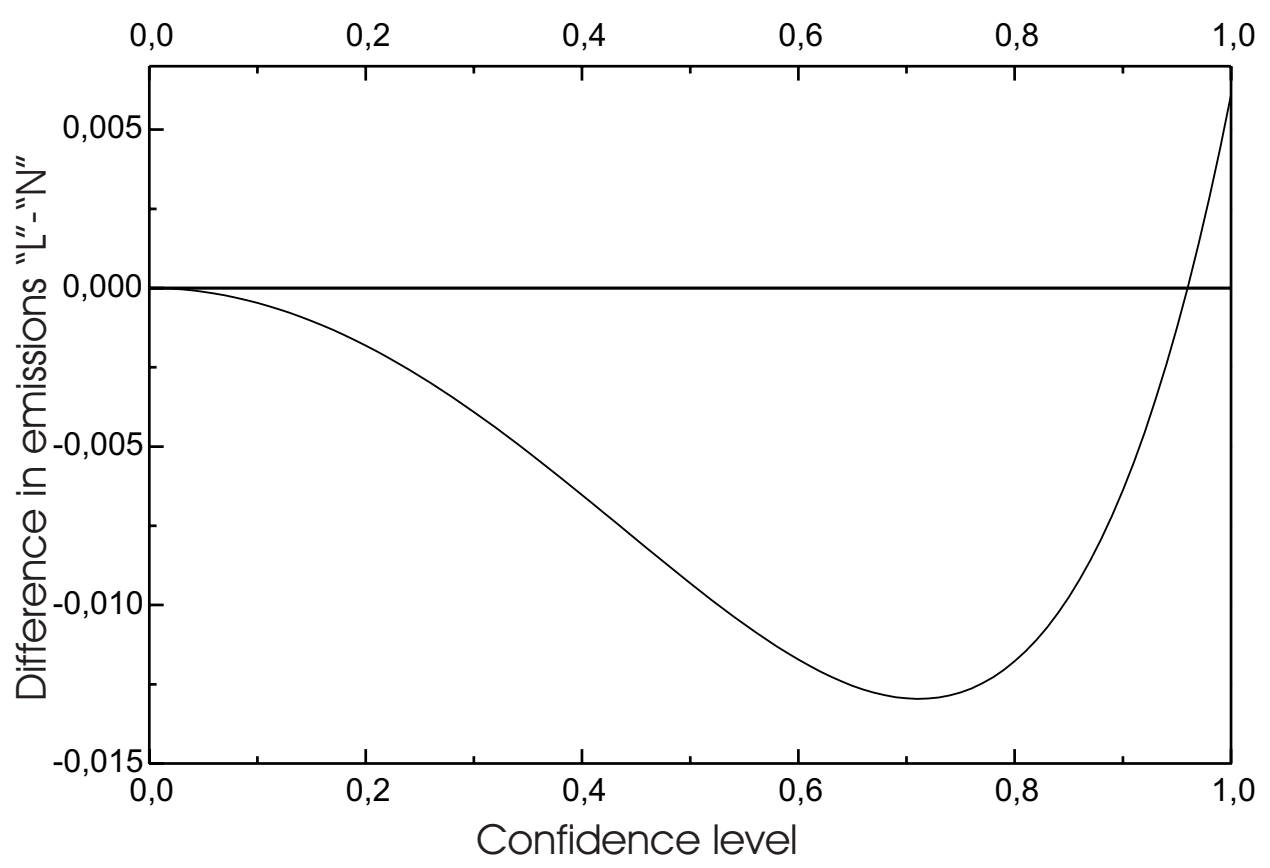

Figure 2: $e_{1}^{L}-e_{1}^{N}$ as a function of the confidence level $0 \leq \lambda \leq 1$.

\subsection{The irreversibility effect and the value of learning}

A critical feature of all decision criteria that are not consistent with EU is that there exist situations where the value of learning is negative (Wakker 1988). This value is 
defined by the difference $W^{L}-W^{N}$ where $W^{x}$ denotes the ex ante (expected) utility for the "learning" and "non-learning" case respectively which can be obtained from (2). Therefore, in a framework where people have an additional choice on whether or not to receive some information, they might choose to stay uniformed. Although being a normative drawback for applying non-EU criteria, this might realistically describe the behavior of some people.

For the model analyzed here, a negative value of learning can occur. In fact, it is related to the irreversibility effect: On the one hand, for $\lambda=1$, i.e. expected utility, the value of learning clearly is positive, the irreversibility effect does not hold. On the other hand, for MaxiMin $(\lambda=0)$, the value of learning is zero and decisions coincide for the " $\mathrm{N}$ " and " $\mathrm{L}$ " case. More generally, the irreversibility effect and the value of learning are connected:

Proposition 2 Let the utility function satisfy assumption 1. Then:

The value of learning is positive if and only if the irreversibility effect does not hold, i.e. the consideration of learning possibilities leads to an increase of emissions $\left(e_{1}^{L}>e_{1}^{N}\right)$.

Thus, if we assume that the behavior of (some) people can be described by the weighted EU and MaxiMin criterion, uncertainty averse people with a low confidence in the probability distribution, could choose not to receive new information on the prospective damages. The reason being, that they would fear getting an information which indicates relative harmlessness of emissions whereas finally severe damages result. Such an information would induce them to choose a higher emission level in period 2 than if learning would not be possible. Thus, if the information cannot be disregarded, they would obviate this potential mistake of building up a too large stock by emitting less in period 1 which leads to the irreversibility effect. However, in a broader framework, where such people have the choice of whether or not to receive information, they would prefer to avoid this thread by staying uniformed. That is, they would not reach the situation in which the irreversibility effect matters.

\section{Conclusions}

In this paper I investigated decisions on abatement of greenhouse gas emissions under uncertainty when based on a weighted average of EU and MaxiMin. Such a decision criterion can be motivated in two different ways: First, because of the fundamental 
uncertainties related to the climate change problem, there is not the single probability distribution. Even if one tries to aggregate different expert's opinions to a single measure one may be attempted to give more weight to the worst case in order to account for unexpected contingencies and to behave cautiously. Second, the failure of expected utility theory to explain behavior of individuals if confronted with fundamental uncertainties motivates the analysis of alternative decision criteria. In this paper, emission levels ere analyzed as a function of the weight attached to the worst case. Thereby, standard results based on expected utility maximization were reconsidered in a broader framework. In particular, the impact of getting new information was studied.

Within a simple two period model that captures some key features of global warming I first showed that a larger weight on the worst case can lead to an increase of emissions. The effect of learning on emissions is not clear in general, but depends qualitatively on the confidence level: For the quadratic utility case, considering prospective learning decreases today's emissions if the weight on EU is small. To put it differently, if the degree of uncertainty (the weight on the worst case) is high, the "irreversibility effect" holds. This finding contrasts the standard results on the irreversibility effect for expected utility maximizer which in our framework corresponds to high confidence levels. Thus, if one behaves more cautiously by giving more weight to the worst case scenario, learning possibilities enhance the importance to pursue a stricter abatement policy today. In a broader framework, however, when people can choose whether or not to receive the (costless) information, it may happen that she decides not to learn because the value of information is negative. For quadratic utility functions this situation coincides with the occurance of the irreversibility effect, i.e. the possibility of learning does not lead to a higher abatement effort because in cases where the irreversibility effect holds, no learning is preferred.

Summarizing, although a decision criterion that gives more weight to the worst case can be motivated in several ways, there are problems of applying it to a dynamic framework at least for normative reasons. A criterion where dynamic inconsistencies imply an increase of emissions when the worst case is weighted more, and where information may have a negative value and thus even costless learning is disregarded, can have merits as a descriptive model but should not serve as a tool for policy advice.

\section{Appendix}

\section{Sufficient conditions for an interior solution for the quadratic case:}


To get an interior solution in period $l, e_{l}$, it suffices to have positive marginal utility at $e_{l}=0$. I.e., for $e_{l}=0: U_{e_{l}}\left(e_{1}, e_{2}, s\right)=a_{l 1}-\left(b_{1}+b_{2} s\right)\left[E_{0}+e_{t}\right]>0(t \neq l)$ for all $e_{t}$ and $s$. The left hand side of this inequality decreases in $s$ and $e_{t}$. We know: $e_{t}<\bar{e}_{t}$, where $\bar{e}_{t}$ denotes the maximal emission level in period $t$, that could be optimal ex post, i.e. the optimal level for $s=0$ and $e_{l}=0$. Therefore, by definition, $U_{e_{1}}\left(\bar{e}_{1}, 0,0\right)=0$ and $U_{e_{2}}\left(0, \bar{e}_{2}, 0\right)=0$. For the quadratic case we obtain $\bar{e}_{t}=\frac{a_{t 1}-b_{1} E_{0}}{a_{t 2}+b_{1}}$. Therefore we have to guarantee that $a_{l 1}-\left(b_{1}+b_{2} s\right)\left(E_{0}+\bar{e}_{t}\right)>0$ for all $s$, i.e.

$$
\begin{aligned}
s<\bar{s} & :=\min _{l \neq t}\left\{\frac{a_{l 1}-b_{1}\left(E_{0}+\bar{e}_{t}\right)}{b_{2}\left(E_{0}+\bar{e}_{t}\right)}\right\} \\
& =\min _{l \neq t}\left\{\frac{\left(a_{l 1}\left(a_{t 2}+b_{1}\right)-b_{1}\left(a_{t 1}+a_{t 2} E_{0}\right)\right.}{b_{2}\left(a_{t 1}+a_{t 2} E_{0}\right)}\right\} .
\end{aligned}
$$

Hereby it is assumed that the parameters guarantee $\bar{s}>0$.

\section{Optimal emission levels for the quadratic case:}

We first derive the optimal second period emission levels given $e_{1}$ for the cases "L" und "N" simultaneously. We define $N(i)=\lambda \int s d \mu(s \mid i)+(1-\lambda) \max s$ for the learning case "L" and $N(i)=\bar{N}:=\lambda \int s d \mu(s)+(1-\lambda) \max s$ for "N". Then, using (5):

$$
e_{2}^{x}\left(e_{1}, i\right)=\frac{a_{21}-\left(b_{1}+b_{2} N(i)\right)\left(E_{0}+e_{1}\right)}{a_{22}+b_{1}+b_{2} N(i)}
$$

where $x=L, N$. Therefore:

$$
\frac{\partial e_{2}^{x}\left(e_{1}, i\right)}{\partial e_{1}}=-\frac{b_{1}+b_{2} N(i)}{a_{22}+b_{1}+b_{2} N(i)} \leq 0 .
$$

Plugging this into (7), we obtain:

$$
\begin{aligned}
0=a_{11}-a_{12} e_{1}+\lambda & \int\left(a_{21}-a_{22} e_{2}^{x}(\cdot)\right) \frac{\partial e_{2}^{x}(\cdot)}{\partial e_{1}} \\
- & {\left[\left(b_{1}+b_{2} s\right)\left(E_{0}+e_{1}+e_{2}^{x}(\cdot)\right]\left(1+\frac{\partial e_{2}^{x}(\cdot)}{\partial e_{1}}\right) d \mu(i, s)\right] } \\
+(1-\lambda)\left[\left(a_{21}-a_{22} \max _{i} e_{2}^{x}(\cdot)\right) \frac{\partial \max _{i} e_{2}^{x}(\cdot)}{\partial e_{1}}\right. & -\left[\left(b_{1}+b_{2} \max s\right)\left(E_{0}+e_{1}+\max _{i} e_{2}^{x}(\cdot)\right]\left(1+\frac{\partial \max _{i} e_{2}^{x}(\cdot)}{\partial e_{1}}\right)\right]
\end{aligned}
$$

Note that

$$
\begin{aligned}
\max _{i} e_{2}^{x}\left(e_{1}, i\right) & =\max _{i} \frac{a_{21}-\left(b_{1}+b_{2} N(i)\right)\left(E_{0}+e_{1}\right)}{a_{22}+b_{1}+b_{2} N(i)} \\
& =\frac{a_{21}-\left(b_{1}+b_{2} \min _{i} N(i)\right)\left(E_{0}+e_{1}\right)}{a_{22}+b_{1}+b_{2} \min _{i} N(i)}
\end{aligned}
$$


For the non-learning case $(x=N)$ we obtain $e_{2}^{N}(\cdot)$ by setting $N(i)=\bar{N}$ for all $i$.

Let us now derive the optimal first period emission levels. For shortness of presentation, define $s^{i}=\int s d \mu(s \mid i)$ and

$$
k(n, s)=\frac{1}{\left(a_{22}+b_{1}+b_{2} n\right)^{2}}\left[\left(b_{1}+b_{2} n\right)^{2}+a_{22}\left(b_{1}+b_{2} s\right)\right]
$$

where $n, s$ are reals. Then, from (13), we obtain for the emission level $e_{1}^{x}(x=L, N)$

$$
e_{1}^{x}=\frac{a_{11}-\left(a_{21}+a_{22} E_{0}\right) K^{x}}{a_{12}+a_{22} K^{x}}
$$

where

$$
K^{x}:=\lambda \int k\left(N(i), s^{i}\right) d \mu(i)+(1-\lambda) k\left(\min _{i} N(i), \max s\right)
$$

and $N(i)=\lambda \int s d \mu(s \mid i)+(1-\lambda) \max s$ for the "L"-case and $N(i)=\bar{N}:=\lambda \int s d \mu(s)+$ $(1-\lambda) \max s$ for $x=N$.

\section{Proof of proposition 1:}

We show that $e_{1}^{L}<e_{1}^{N}$ for small $\lambda$. Using (16), it suffices to show that $K^{L}>K^{N}$ for small $\lambda$. We show that (a) $e_{1}^{L}$ and $e_{1}^{N}$ and (b) the derivatives w.r.t $\lambda$ coincide at $\lambda=0$. We therefore study the second derivatives which are derived in (c) and then compared in $(\mathrm{d})$.

(a)

For $\lambda=0$ it follows for the "L"-case $N(i)=\lambda \int s d \mu(s \mid i)+(1-\lambda) \max s=\max s$ and for $x=N$ we get $\bar{N}=N(i)=\lambda \int s d \mu(s)+(1-\lambda) \max s=\max s$. Remembering (17), we immediately obtain $K^{N}=K^{L}$.

(b)

To prepare the proof, consider first the partial derivatives of $k(n, s)$, defined in (14):

$$
\begin{aligned}
\frac{\partial k}{\partial n} & =\frac{2 a_{22} b_{2}^{2}}{\left(a_{22}+b_{1}+b_{2} n\right)^{3}}(n-s) \\
\frac{\partial k_{1}}{\partial s} & =\frac{a_{22} b_{2}}{\left(a_{22}+b_{1}+b_{2} n\right)^{2}} \geq 0
\end{aligned}
$$

where $\frac{\partial k}{\partial n}>0(<0)$ if $n>s(n<s)$.

Note that

$$
\frac{d K^{x}}{d \lambda}=\frac{\partial K^{x}}{\partial \lambda}+\sum_{i} \frac{\partial K^{x}}{\partial N(i)} \frac{d N(i)}{d \lambda}
$$


Here, using (17) it follows immediately

$$
\frac{\partial K^{x}}{\partial \lambda}=\int k\left(N(i), s^{i}\right) d \mu(i)-k\left(\min _{i} N(i), \max s\right) .
$$

Noting that $N(i)=\max s$ at $\lambda=0$ for all $i$ for both the "learning" and the "nonlearning" -case, we immediately see that $\frac{\partial K^{L}}{\partial \lambda}=\frac{\partial K^{N}}{\partial \lambda}$ at $\lambda=0$.

Further, let us define $\delta_{i}=1$ if $i=i *:=\arg \min _{j} N(j)$ and $\delta_{i}=0$ otherwise. Then, using (18) and (19) and $N(i)=\max s$ at $\lambda=0$, we obtain for the derivatives of (17) w.r.t. $N(i)$ :

$$
\begin{aligned}
\frac{\partial K^{x}}{\partial N(i)} & =\lambda \mu(i) \frac{\partial k\left(N(i), s^{i}\right)}{\partial n}+\delta_{i}(1-\lambda) \underbrace{\frac{\partial k(N(i), \max s)}{\partial n}}_{=0 \text { for } \lambda=0} \\
& =0 \text { for } \lambda=0
\end{aligned}
$$

Thus, at $\lambda=0, \frac{d K^{L}}{d \lambda}=\frac{d K^{N}}{d \lambda}$.

(c)

We now derive the second derivatives $\frac{d^{2} K^{L}}{d \lambda^{2}}$ and $\frac{d^{2} K^{N}}{d \lambda^{2}}$. Here, with (20) and (21) we obtain at $\lambda=0$ :

$$
\begin{aligned}
\frac{d^{2} K^{L}}{d \lambda^{2}} & =\underbrace{\frac{\partial^{2} K^{L}}{(\partial \lambda)^{2}}}_{=0}+2 \sum_{i} \frac{\partial^{2} K^{L}}{\partial \lambda \partial N(i)} \frac{d N(i)}{d \lambda}+\sum_{i} \frac{\partial^{2} K^{L}}{(\partial N(i))^{2}}\left(\frac{d N(i)}{d \lambda}\right)^{2}+\sum_{i} \underbrace{\frac{\partial K^{L}}{\partial N(i)}}_{=0 \text { for } \lambda=0} \frac{d^{2} N(i)}{d \lambda^{2}} \\
& =2 \sum_{i} \frac{\partial^{2} K^{L}}{\partial \lambda \partial N(i)} \frac{d N(i)}{d \lambda}+\sum_{i} \frac{\partial^{2} K^{L}}{(\partial N(i))^{2}}\left(\frac{d N(i)}{d \lambda}\right)^{2} .
\end{aligned}
$$

Thus, we have to study $\frac{\partial^{2} K^{L}}{\partial \lambda \partial N(i)}$ and $\frac{\partial^{2} K^{L}}{(\partial N(i))^{2}}$. Taking into account (18), partial differentiation of (20) w.r.t. $N(i)$ gives at $\lambda=0$ :

$$
\begin{array}{rll}
\frac{\partial^{2} K^{L}}{\partial \lambda \partial N(i)} & = & \mu(i) \frac{\partial k\left(N(i), s^{i}\right)}{\partial n}-\delta_{i} \frac{\partial k(N(i), \max s)}{\partial n} \\
& \left.=\quad \mu(i) T(N(i))\left(N(i)-s^{i}\right)-\delta_{i} N(i)-\max s\right) T(N(i)) \\
& \stackrel{N(i)=\max s}{=} & \mu(i) T(\max s)\left(\max s-s^{i}\right)
\end{array}
$$

where $T(N(i))=\frac{2 a_{22} b_{2}^{2}}{\left(a_{22}+b_{1}+b_{2} N(i)\right)^{3}}$ and again $\delta_{i}=1$ if $i=i *:=\arg \min _{j} N(j)$ and $\delta_{i}=0$ otherwise.

Further, from (18) and (21) we obtain at $\lambda=0$ :

$$
\frac{\partial^{2} K^{L}}{(\partial N(i))^{2}}=\delta_{i} \frac{\left.\partial^{2} k(N(i), \max s)\right)}{\partial n^{2}}
$$




$$
\begin{aligned}
& =\delta_{i}[T(N(i))+\frac{T(N(i))}{a_{22}+b_{1}+b_{2} N(i)} \underbrace{(N(i)-\max s)}_{=0}] \\
& =\delta_{i} T(\max s)
\end{aligned}
$$

Plugging these partial derivatives into (22), we finally arrive at

$$
\begin{aligned}
\frac{d^{2} K^{L}}{d \lambda^{2}} & =T(\max s)[2 \sum_{i} \mu(i)\left(\max s-s^{i}\right) \underbrace{\frac{d N(i)}{d \lambda}}_{=s^{i}-\max s}+(\underbrace{\left.\frac{d N(i *)}{d \lambda}\right)^{2}}_{=s^{i *}-\max s}] \\
& =T(\max s)\left[-2 \sum_{i}\left[\mu(i)\left(\max s-s^{i}\right)^{2}\right]+\left(\max s-s^{i *}\right)^{2}\right]
\end{aligned}
$$

for the "L"-case at $\lambda=0$.

Changing $s^{i}$ and $s^{i *}$ into $\int s d \mu(i, s)=\sum \mu(i) s^{i}$, we directly obtain the partial derivative for the case $x=N$ :

$$
\frac{d^{2} K^{N}}{d \lambda^{2}}=-T(\max s)\left[\max s-\sum_{i} \mu(i) s^{i}\right]^{2}
$$

Thus, $\frac{d^{2} K^{L}}{d \lambda^{2}}>\frac{d^{2} K^{N}}{d \lambda^{2}}$ at $\lambda=0$ is equivalent to

$$
\left(\max s-\sum \mu(i) s^{i}\right)^{2}-2 \sum_{i}\left[\mu(i)\left(\max s-s^{i}\right)^{2}\right]+\left(\max s-s^{i *}\right)^{2}>0 .
$$

(d)

Let us denote $d^{i}=\max s-s^{i}$ and w.l.o.g. we assume that $d^{1} \geq d^{2} \geq \ldots$, i.e. $i *=1$. Then we have to show that

$$
\Delta:=\left(\sum_{i} \mu(i) d^{i}\right)^{2}-2 \sum_{i} \mu(i)\left(d^{i}\right)^{2}+\left(d^{1}\right)^{2}>0
$$

Now defining $\pi(2)=\mu(1)+\mu(2)$ and $\pi(i)=\mu(i)$ for $i \geq 3$, simple transformations lead to:

$$
\begin{aligned}
& \Delta=\left(\sum_{i \geq 2} \pi(i) d^{i}\right)^{2}-2 \sum_{i \geq 2} \pi(i)\left(d^{i}\right)^{2}+\left(d^{2}\right)^{2} \\
& +\underbrace{\left[d^{1}-d^{2}\right]\left[\left(d^{1}+d^{2}\right)\left(1-2 \mu(1)+\mu(1)^{2}\right)+2 \mu(1) \sum_{i \geq 2} \mu(i) d^{i}\right]}_{\geq 0} \\
& \geq\left(\sum_{i \geq 2} \pi(i) d^{i}\right)^{2}-2 \sum_{i \geq 2} \pi(i)\left(d^{i}\right)^{2}+\left(d^{2}\right)^{2}
\end{aligned}
$$


with strict inequality if $d^{1}>d^{2}$. Repeating this procedure we get $\Delta \geq 0$. Thus, $\frac{d^{2} K^{L}}{d \lambda^{2}}>\frac{d^{2} K^{N}}{d \lambda^{2}}$, if not all $d^{i}\left(s^{i}\right)$ coincide. Therefore, it finally follows that $K^{L}>K^{N}$ for small $\lambda$ and the "N" emission level exceeds the emissions when learning is taken into account. Q.E.D.

\section{Proof of proposition 2:}

Let us now compare the expected ex ante utility for "no-learning", $W^{N}$, and "learning", $W^{L}$. Under assumption 1) we obtain:

$$
\begin{aligned}
V^{1}\left(e_{1}\right) & =-\frac{1}{2 a_{12}}\left(a_{11}-a_{12} e_{1}\right)^{2} \\
e_{2}^{x}\left(e_{1}, i\right) & =\frac{a_{21}-\left(b_{1}+b_{2} N(i)\right)\left(E_{0}+e_{1}\right)}{a_{22}+b_{1}+b_{2} N(i)} \\
e_{1}+e_{2}^{x}\left(e_{1}, i\right) & =\frac{a_{21}+a_{22} e_{1}-\left(b_{1}+b_{2} N(i)\right) E_{0}}{a_{22}+b_{1}+b_{2} N(i)} \\
V^{2}\left(e_{2}^{x}\left(e_{1}, i\right)\right) & =-\frac{1}{2 a_{22}}\left(a_{21}-a_{22} e_{2}^{x}\left(e_{1}, i\right)\right)^{2} \\
& =-\frac{a_{21}+a_{22}\left(E_{0}+e_{1}\right)}{2 a_{22}}\left(\frac{\left(b_{1}+b_{2} N(i)\right.}{a_{22}+b_{1}+b_{2} N(i)}\right)^{2}
\end{aligned}
$$

Thus, the ex ante payoff $W^{x}$ is given by

$$
\begin{aligned}
W^{x}= & V^{1}\left(e_{1}^{x}\right)+\lambda \int V^{2}\left(e_{2}^{x}\left(e_{1}^{x}, i\right)\right)-D\left(e_{1}^{x}+e_{2}^{x}\left(e_{1}^{x}, i\right), s\right) d \mu(i, s) \\
& +(1-\lambda)\left[V^{2}\left(e_{2}^{x}\left(e_{1}^{x}, i *\right)\right)-D\left(e_{1}^{x}+e_{2}^{x}\left(e_{1}^{x}, i *\right), \max s\right)\right] \\
= & -\frac{\left(a_{11}-a_{12} e_{1}^{x}\right)^{2}}{2 a_{12}}-\frac{a_{21}+a_{22}\left(E_{0}+e_{1}^{x}\right)}{2 a_{22}}\left[\lambda \int \frac{\left(b_{1}+b_{2} N(i)\right)^{2}+\left(b_{1}+b_{2} s\right) a_{22}}{\left(a_{22}+b_{1}+b_{2} N(i)\right)^{2}} d \mu(i, s)\right. \\
& \left.+(1-\lambda) \frac{\left(b_{1}+b_{2} N(i *)\right)^{2}+\left(b_{1}+b_{2} \max s\right) a_{22}}{\left(a_{22}+b_{1}+b_{2} N(i *)\right)^{2}}\right] \\
= & -\frac{\left(a_{11}-a_{12} e_{1}^{x}\right)^{2}}{2 a_{12}}-\frac{\left(a_{21}+a_{22}\left(E_{0}+e_{1}^{x}\right)\right)^{2}}{2 a_{22}} K^{x} \\
\stackrel{(16)}{=}- & -\frac{\left(a_{11} a_{22}+a_{12} a_{21}+a_{12} a_{22} E_{0}\right)^{2}}{2 a_{12} a_{22}} \frac{K^{x}}{\left(a_{12}+a_{22} K^{x}\right)}
\end{aligned}
$$

Thus, we see that $W^{x}$ is decreasing in $K^{x}$. Therefore $W^{L}>(<) W^{N}$ if and only if $K^{L}<(>) K^{N}$ : Combining this with $e_{1}^{L}>(<) e_{1}^{N}$ if and only if $K^{L}<(>) K^{N}$, completes the proof. Q.E.D.

\section{References}

Aaheim, H.A. and C. Bretteville (2001): Decision-making frameworks for climate policy under uncertainty, CICERO Working Paper 2001:2, University of Oslo. 
Arrow, K.J. and A.C. Fisher (1974): Environmental preservation, uncertainty and irreversibility, Quarterly Journal of Economics 88, 312-319.

Bretteville, C. (1999): Decision criteria under uncertainty and the climate problem, CICERO Working Paper 1999:10, University of Oslo.

Camerer, C. and M. Weber (1992): Recent developments in Modeling preferences: Uncertainty and ambiguity, Journal of risk and uncertainty 5, 325-370.

Chichilnisky, G. (2000): An axiomatic aproach to choice under uncertainty with catastrophic risks, Resource and Energy Economics 22, 221-231.

Chichilnisky, G. and G.M.Heal (1993): Global environmental risks, Journal of Economic Perspectives 7, 65-86.

Eichberger, J. and D. Kelsey (1997):Signalling games with Uncertainty, Paper presented at ESEM98.

Ellsberg, D. (1961): Risk Ambiguity and the Savage Axioms, Quarterly Journal of Economics 75, 643-669.

Epstein, L.G. (1980): Decision making and the temporal resolution of uncertainty, International Economic Review 21, 269-283.

Epstein, L.G. und M. le Breton (1993): Dynamically consistent beliefs must be Bayesian, Journal of Risk and Uncertainty 61, 1-22.

Gilboa, I. (1987): Expected utility with purely subjective non-additive probabilities, Journal of Mathematical Economics 16, 65-88.

Henry, C. (1974): Investment decisions under uncertainty: the irreversibility effect, American Economic Review 64, 1006-12.

IPCC III (1996): Climate Change 1995 - Economic and Social Dimensions of Climate Change: Contributions of Working Group III to the second assessment report of the IPCC, Bruce, J.P., H. Lee und E.F. Haites (Hrsg.), Cambridge University Press: Cambridge.

Karni, E. and Z. Safra (1989): Ascending bid auctions with behaviorally consistent bidders, Annals of Operations Research 19, 435-446.

Machina, M.J. (1989): Dynamic consistency and non-expected utility models of choice under uncertainty, Journal of Economic Literature 27, 1622-1668.

Morgan, M.G. and D.W. Keith (1995): Subjective Judgments by Climate Experts, Environmental Science and Technology 29, 468A-476A. 
Schmeidler, D. (1989): Subjective probability and expected utility without additivity, Econometrica 57, 571-87.

Ulph, A. and D. Ulph (1997): Global warming, irreversibility and learning, The Economic Journal 107, 636-650.

Wakker, P.P. (1988): Nonexpected utility as aversion to information, Journal of Behavioral Decision Making 1, 169-175. 